\title{
CONHECIMENTO DO CLIENTE DIABÉTICO EM RELAÇÃO AOS CUIDADOS COM OS PÉS*
}

\author{
KNOWLEDGE ABOUT FEET CARE OF THE DIABETIC CLIENT
}

\section{CONOCIMIENTO DE LOS DIABÉTICOS SOBRE LOS CUIDADOS CON LOS PIES}

\author{
Elaine Cristina Barbui** \\ Maria Inês Monteiro Cocco***
}

Barbui EC, Cocco MIM. Conhecimento do clinte diabético em relação aos cuidados com os pés. Rev Esc Enferm USP 2002; 36(1): 97-103.

\section{RESUMO}

Esta pesquisa tem por objetivo avaliar o conhecimento dos clientes que freqüentam um Ambulatório de Diabetes, em relação à sua doença e cuidados com os pés. Na amostra estudada-diabéticos tipo 2 - houve predominância da faixa etária acima de cinqüenta anos e 71,8\% tinham diabetes há menos de dez anos. Concluiu-se que os clientes sabem que os cuidados adequados com os pés são necessários para evitar as complicações, porém o autocuidado não é realizado corretamente. Para que isso seja incorporado em seu cotidiano, é fundamental que tenham acesso às informações sobre diabetes, participação da família, além de habilidade e motivação.

PALAVRAS-CHAVE: Diabetes mellitus. Pé diabético. Educação em saúde. Enfermagem em Saúde Pública.

\section{ABSTRACT}

The objective of this research is to evaluate how much the clients that frequently went to the Diabetes Ambulatory, knew about their own ill, and the feet care. In the sample - diabetes type 2 - the majority were people with more than fifty years old and $71.8 \%$ were diabetics for more than ten years. So the conclusion that could be taken is that the clients $\mathrm{knew}$ about the care that they must have with their feet to don't have complications, but the "own care" is not well done. To insert it in the rightly on their day-by-day they must have acess to the information about diabetes, participation of the family, motivation and their own hability.

KEYWORDS: Diabetes mellitus. Diabetic foot. Health education. Public health nursing.

\section{RESUMEN}

Este estudio tuvo por objetivo evaluar el conocimiento de los diabeticos tipo 2 atendidos en uno Ambulatorio de Diabetes en relación a su enfermedad y los cuidados con los pies. La major faja de edad fue más de cincuenta años y $71,8 \%$ teniam diabetes ha mas de diet años. Los resultados no permitieron concluir que los diabeticos saben que los cuidados adecuados con los pies son necesarios hacia evitar las complicaciónes, pero el atuocuidado no es realizado correctamente. Para incorporar esto en su cotidiano es fundamental que los diabeticos tengam acceso a las informaciones sobre diabetes, allende la participación de la familia, habilidad y motivación.

PALABRAS-CLAVE: Diabetes mellitus. Pie diabético. Educación en salud. Enfermagem en Salud Publica.

\footnotetext{
Projeto de pesquisa financiado pelo SAE (Serviço de Apoio ao Estudante). Universidade Estadual de Campinas UNICAMP

** Enfermeira - Campinas. SP

*** Professora Assistente Doutora. Departamento de Enfermagem. FCM-UNICAMP. Coordenadora do Grupo de Estudos e Pesquisas em Saúde e Trabalho. Membro do Laboratório de Estudos e Pesquisas em Práticas de Educação e Saúde PRAESA, Faculdade de Educação / UNICAMP. inesmon@fcm.unicamp.br
} 


\section{INTRODUÇÃO}

O diabetes é uma síndrome metabólica de grande importância na atualidade. No Brasil há aproximadamente cinco milhões de diabéticos e 50\% deles não conhecem o diagnóstico. Para o tratamento do diabetes tipo 20 hipoglicemiante oral é usado por $40 \%$ e a insulina por $8 \%$ dos diabéticos. Se o excesso de peso fosse evitado, $50 \%$ dos novos casos poderiam ser evitados, assim como haveria uma diminuição de $30 \%$, com a realização de atividade física. Através do controle da pressão arterial podem ser reduzidos "... $80 \%$ dos acidentes vasculares cerebrais, $60 \%$ das amputações de membros inferiores, 50\% das doenças renais terminais e 40\% das doenças coronarianas". Os programas educativos para os diabéticos e seus familiares têm um importante papel na melhora da qualidade de vida, assim como na redução das hospitalizações por diabetes(1).

A freqüência do diabetes vem aumentando nos últimos anos, tanto nos países desenvolvidos como nos países em desenvolvimento. Isso ocorre devido ao aumento da sobrevida do diabético, que é favorecida pelos melhores cuidados, mudança do estilo de vida, favorecendo os fatores de risco para o diabetes, assim como pelo aumento da esperança de vida de pessoas de maior risco.

O diabetes mellitus é uma das doenças crônicas com maior prevalência atualmente em diversos paises e vários fatores têm contribuído para que isso ocorra: maior taxa de urbanização, industrialização, sedentarismo, obesidade e dietas hipercalóricas e ricas em hidratos de carbono de absorção rápida(2).

São complicações crônicas do diabetes a retinopatia, presente em metade dos diabéticos após 10 anos de doença e $60-80 \%$ após 15 anos ou mais; a nefropatia está presente em 5 a $10 \%$ dos diabéticos tipo 2, após 20 anos de doença; a macroangiopatia, que engloba a cardiopatia isquêmica, a doença cerebrovascular e a doença vascular periférica, além da neuropatia. A neuropatia é uma das formas mais freqüentes de complicação da doença, sendo constatada em 8 a $12 \%$ dos diabéticos tipo 2 (ao ser feito o diagnóstico da doença) e também após longos períodos do início da doença, 20-25 anos, em 50-60\% dos diabéticos. Em relação às alterações nos pés dos diabéticos, $10 \%$ têm ulceração nos pés durante a evolução da doença; 20 a 25\% das internações dos diabéticos estão relacionadas às complicações nos pés(1)

Este quadro pode ser revertido pois "a aplicação dos conhecimentos e recursos existentes, de forma sistemática tem produzido uma redução na morbidade, mortalidade e custos relativos ao diabetes. As intervenções podem ser voltadas à prevenção de fatores de risco de desenvolvimento do diabetes e do surgimento de complicações". Tais medidas, se estimadas para os EUA, levariam a uma diminuição considerável de 50\% das amputações; caso fossem estimadas para o Brasil, esse impacto poderia ser ainda maior. Este é um dado importante pois implica na qualidade de vida do diabético, além do custo financeiro( ${ }^{3)}$. Entre as complicações crônicas, as ulcerações dos pés e amputações poderiam ser evitadas, com informações adequadas e a realização de exames(4))

A American Diabetes Association recomenda a identificação dos fatores de risco que estão associados à elevação no risco de amputação, que são a "... neuropatia periférica, evidência de aumento da pressão - calos, hematomas - mobilidade limitada da articulação, deformidade óssea, ou patologias severas nas unhas, doença vascular periférica e história [anterior] de úlceras ou amputação"(5).

$\mathrm{Na}$ tentativa de proporcionar melhoria na qualidade de vida do diabético e prevenção das complicações, é necessário elaborar um plano assistencial que vise assistir e educar o cliente ${ }^{(6)}$. As ações educativas são fundamentais considerando-se que pouco menos da metade $(45 \%)$ das pessoas diabéticas e $1 / 3$ das pessoas com mais de vinte anos de doença desenvolverão doença vascular periférica e, provavelmente, evoluirão para gangrenas e amputações, traumas estes que poderiam ser evitados, se fossem tomados os cuidados apropriados com os pés ${ }^{(7)}$.

Deve ser destacado ainda que no decorrer da evolução do diabetes, $10 \%$ dos pacientes têm ulceração nos pés; 20 a 25\% das internações ocorrem devido a complicações nos pés. Das amputações não traumáticas de membros inferiores, metade ocorre nos diabéticos ${ }^{(1)}$.

Os principais sintomas da neuropatia periférica são inicialmente a parestesia (formigamento) e sensação de queimação (especialmente à noite) e à medida que a neuropatia progride, ocorre a perda da sensibilidade. Os clientes com neuropatia têm maior risco para o surgimento de danos e infecções não detectados nos pés devido à diminuição das sensações de dor e temperature(8).

A maioria dos casos de amputações ocorre em clientes diabéticos que não tinham recebido orientações sobre os cuidados com os pés, ou que não tinham seguido as mesmas adequadamente. Na grande maioria das vezes culpa-se o cliente pelas elevadas estatísticas de amputações em diabetes, porém, é comum encontrar clientes com úlceras avançadas nos pés e que poucas semanas antes haviam ido ao médico e perguntado se havia algum problema com os seus pés. O médico limitou-se ao que o cliente acreditou estar correto. Tem havido sérias deficiências na forma como o profissional de saúde vem examinando o diabético e, especificamente, o exame adequado nos pés e informações adequadas( ${ }^{(4)}$. 
Os pés de pessoas com diabetes podem apresentar complicações que se manifestam através de três formas clinicas: isquemia aguda (trombose arterial); infecção aguda - gangrena úmida; infecção crônica - necrose de partes moles e osteomielite.

Numa consulta de rotina para o cliente diabético deve-se levar em conta os seguintes fatores de risco: idade superior a 40 anos; tabagismo; diabetes com mais de 10 anos de duração; diminuição dos pulsos arteriais ou hipoestesia em membros inferiores; deformidades anatômicas (artropatia, calosidades); presença de ulcerações ou amputações prévias. Os clientes devem ser orientados a examinar seus pés, visando a detecção precoce de possiveis complicações, assim como seu tratamento, quando necessário.

Concordamos que "a educação em saúde, por sua vez, é uma forma concreta de apontar várias possibilidades aos usuários, abandonando a premissa do caminho único, dos dogmas do saber na área de saúde. Uma forma de adquirir esta autonomia nas relações profissional - clientela, é através da possibilidade de uma educação libertadora, na qual assume seu lugar como agente do processo, com poder de decisão, pelo próprio conhecimento que tem da realidade"(9).

O enfermeiro tem um papel fundamental na realização de atividades de educação e saúde junto ao diabético e seus familiares. A consulta deve ser integrada por profissionais de saúde de diferentes áreas, atuando como grupo multidisciplinar, tendo como elemento principal o cliente diabético ${ }^{(10)}$

As ações educativas realizadas com os clientes diabéticos têm sido voltadas somente para o controle glicêmico, não sendo, em geral, considerados os aspectos psicológicos, sociais, culturais e de relacionamento, levando a uma baixa aderência dos clientes diabéticos em relação ao autocuidado(2).

Concordamos que "as práticas de educação e saúde deveriam estar voltadas, numa perspectiva mais democrática, para um processo de formação de cidadania e desenvolvimento da consciência sanitária e que o cliente torne-se sujeito deste processo, que deve ter como referência a apropriação de conhecimentos tanto pelo educador, quanto pelo educando"(11)

Através da observação pessoal dos clientes diabéticos que freqüentavam o Ambulatório de Diabetes e especificamente o Ambulatório de Pé Diabético de um hospital universitário, no interior do Estado de São Paulo, foi detectada a necessidade de desenvolvimento deste estudo, pois os mesmos apresentavam complicações devido à sua doença, sendo que estas, em grande parte, poderiam ser evitadas se os mesmos tivessem informações adequadas e conhecimento a respeito de sua doença e os cuidados a serem tomados para evitar as complicações crônicas e agudas, principalmente as que causam a amputação dos membros e pés.

\section{OBJETIVO}

Avaliar o conhecimento dos clientes diabéticos em relação à sua doença e, especificamente, em relação aos cuidados adequados com os pés.

\section{METODOLOGIA}

A população estudada constituiu-se de clientes do Ambulatório de Diabetes e do Ambulatório de Pé Diabético, com diabetes tipo 2 , atendidos em um hospital universitário público, que aguardavam a realização da consulta e concordaram em responder às perguntas formuladas.

Foram entrevistadas 32 pessoas no período de julho a setembro de 1997, aleatoriamente, enquanto aguardavam atendimento no Ambulatório de Diabetes, além dos clientes que apresentavam complicações nos pés e estavam sendo atendidos no Ambulatório de Pé Diabético.

$\mathrm{Na}$ metodologia de pesquisa utilizada, a amostragem "privilegia os sujeitos sociais que detêm os atributos que o investigador pretende conhecer; considera-os em número suficiente para permitir uma certa reincidência das informações, porém não despreza informações impares cujo potencial explicativo tem que ser levado em conta"(12).

O instrumento utilizado para a coleta de dados foi um questionário, aplicado na sala de espera do Ambulatório; foram realizadas seis entrevistas para o pré-teste, com os clientes diabéticos do tipo 2 acompanhados neste serviço.

$\mathrm{O}$ instrumento para coleta de dados contém duas partes, sendo a primeira referente aos dados de identificação, condições de saúde, atividade física e condições de moradia. A segunda parte contém dados específicos em relação aos cuidados com os pés: exame dos pés na residência, cuidados específicas, tipo de calçado utilizado e alterações na sensibilidade.

Em relação aos aspectos éticos, foi obtido o consentimento informado dos entrevistados.

\section{RESULTADOS}

\section{CARACTERÍSTICAS DA AMOSTRA}

\section{Dados sociodemográficos}

$\mathrm{Na}$ amostra estudada, 19 pessoas eram do sexo feminino $(59,4 \%)$ e 13 , do sexo masculino $(40,6 \%)$. 
Tabela 1 - Distribuição da amostra por faixa etária. Campinas, 1998.

\begin{tabular}{ccc}
\hline Idade (anos) & Freqüência & Percentual (\%) \\
\hline 40 a 49 & 04 & 12,5 \\
50 a 59 & 14 & 43,8 \\
60 a 69 & 06 & 18,7 \\
70 a 79 & 08 & 25,0 \\
\hline Total & 32 & 100,0 \\
\hline
\end{tabular}

A faixa etária predominante $(43,8 \%)$ foi a de 50 a 59 anos, seguida pela de 70 a 79 anos, com $25 \%$ (tabela I). Na amostra estudada $75 \%$ dos clientes eram casados; $15,6 \%$ viúvos e $9,4 \%$ solteiros.

Tabela 2 - Distribuição da amostra segundo a escolaridade. Campinas, 1998.

\begin{tabular}{lcc}
\hline \multicolumn{1}{c}{ Escolaridade } & $\begin{array}{c}\text { Fre- } \\
\text { qüência }\end{array}$ & $\begin{array}{c}\text { Percen- } \\
\text { tual (\%) }\end{array}$ \\
\hline Analfabeto & 06 & 18,8 \\
Ensino fundam. incompleto & 21 & 65,6 \\
Ensino médio incompleto & 02 & 6,25 \\
Ensino médio completo & 01 & 3,1 \\
Ensino superior & 02 & 6,25 \\
\hline Total & 32 & 100,00 \\
\hline
\end{tabular}

Em relação à escolaridade, $18,8 \%$ eram analfabetos e $65,6 \%$ tinham o ensino fundamental incompleto (tabela II).

Da amostra estudada 53,1\% era aposentada e o restante, $46,9 \%$, não recebia aposentadoria. Entre os aposentados somente uma pessoa exercia outro tipo de atividade remunerada.

Em relação às condições de moradia, 87,5\% (28 pessoas) tinham residência própria, uma morava em casa cedida e somente $9,4 \%$ moravam em casas alugadas.

Quanto ao grau de parentesco que os clientes diabéticos tinham em relação às pessoas com quem moravam, 40,6\% residiam com o cônjuge e filhos; $31,3 \%$ com o cônjuge; $12,5 \%$ com os filhos e $3,1 \%$, com outras pessoas, totalizando $87,5 \%$ dos mesmos (28 pessoas) que moravam com algum familiar, ou alguma pessoa que lhes faziam companhia; somente $12,5 \%$ (quatro pessoas), moravam sozinhos.

$\mathrm{Na}$ residência da maioria dos entrevistados $(46,9 \%)$, o piso era de cerâmica; $15,6 \%$, taco e cerâmica; em 12,5\% o chão era cimentado; em 9,4\% era taco, semelhante ao número de cerâmica. Em 3,1\% das residências o piso era de vermelhão e cerâmica, valor semelhante ao taco, vermelhão e cerâmica.
Em relação ao tipo de quintal em $46,9 \%$ das residências era cimentado; em $25 \%$, de terra; em 15,6\% parcialmente terra e cimentado, e em $12,5 \%$, não havia quintal.

\section{Dados relativos ao diabetes}

Em relação à distribuição da amostra de acordo com o tempo de diabetes, $71,9 \%$ dos entrevistados tinham até dez anos de doença, 25\% entre 11 e 20 anos e uma pessoa não soube responder.

A maioria do entrevistados, 96,9\%, fazia uso de hipoglicemiantes orais e/ou insulina para o controle de sua doença, $46,9 \%$ usavam hipoglicemiantes orais; $37,5 \%$ insulina e $12,5 \%$ utilizavam ambos, concomitantemente. Somente uma pessoa $(3,1 \%)$ não utilizava medicações.

Em relação a alteração na visão, 40,6\% dos clientes entrevistados referiram ter problemas de visão relacionados ao diabetes, mas, somente quatro souberam dizer quais eram essas complicações: dois tinham retinopatia diabética e dois, catarata.

Com referência à prática de atividade física, 13 pessoas realizavam algum tipo de atividade $(40,6 \%)$, enquanto 19 pessoas disseram não praticar nenhum tipo de exercício físico $(59,4 \%)$. Dentre as atividades fisicas referidas 11 pessoas faziam caminhada $(34,4 \%)$; uma fazia caminhada e alongamento $(3,1 \%)$ e outra, caminhada e natação $(3,1 \%)$.

\section{Dados referentes aos cuidados com os pés}

Em relação aos cuidados com os pés, $81,3 \%$ responderam afirmativamente à questão sobre o cuidado adequado dos pés ser um fator importante na prevenção de complicações decorrentes do diabetes. Um dos clientes que respondeu afirmativamente a esta questão, disse que só sabia porque havia tido uma complicação nos pés, embora fosse diabético há vinte anos. Entre as seis pessoas $(18,8 \%)$ que responderam negativamente, uma era diabética há menos de dez anos, outra há quinze anos, porém demonstrava desinformação sobre a doença.

O exame dos pés era realizado por $65,6 \%$ dos entrevistados, mas, somente $50 \%$ dos entrevistados examinavam os pés diariamente. As justificativas mais freqüentes referidas pelos entrevistados foram: diabetes há menos de um ano; só examina quando vai ao Ambulatório de Diabetes; nunca tem nada nos pés, não acredita nesta necessidade e já tem 69 anos. Outra pessoa relatou que não examinava os pés devido às dificuldades inerentes à idade, 71 anos, o que dificultava a realização do exame e seus familiares não o faziam. 
Tabela 3 - Distribuição da amostra de acordo com o tipo de sapato usado. Campinas, 1998.

\begin{tabular}{lcc}
\hline \multicolumn{1}{c}{ Tipo de sapato } & $\begin{array}{r}\text { Fre- } \\
\text { qüência }\end{array}$ & $\begin{array}{c}\text { Percen- } \\
\text { tual (\%) }\end{array}$ \\
\hline Sapatos fechados & 20 & 62,5 \\
Sapatos abertos & 03 & 9,4 \\
Sapatos fechados e abertos & 09 & 28,1 \\
\hline Total & 32 & 100,0 \\
\hline
\end{tabular}

Em relação aos cuidados com os pés durante e após o banho, 15,6\% responderam que lavam com água morna e sabonete e enxugam, sem cuidados especificos. Duas dessas pessoas responderam que sabiam que os cuidados adequados eram necessários para.prevenir as complicações e três não sabiam quais os cuidados adequados; $84,4 \%$ referiram lavar com água e sabonete, enxugando-os adequadamente.

A verificação diária da temperatura, cor e locais de pressão nos pés era realizada por somente $37,5 \%$ dos entrevistados. Apesar de receberem informações pertinentes ao uso adequado de calçados, 18,8\% referiram que andavam descalços em casa.

A maioria dos entrevistados $(68,8 \%)$ examinava sempre dentro de seus sapatos para verificar se havia objetos estranhos dentro deles.

Deve ser destacada a ausência de preocupação em relação ao conforto proporcionado pelos sapatos (não apertar, não incomodar) para 90,6\% dos entrevistados; 9,4\% referiram usar calçados apertados.

Todos os entrevistados tinham conhecimento de que o fumo é um fator de risco para o desenvolvimento das complicações diabéticas, especialmente para os pés, embora $12,5 \%$ continuassem fumando, sendo que um deles durante 42 anos.

No cuidado com as lesões e/ou calos nos pés $40,6 \%$ das pessoas referiram que quando tinham alguma lesão nas pernas / pés ou calos, tratavam em casa fazendo uso de anti-séptico, iodo, mercúrio, álcool ou pomada.

- 10 pessoas só disseram que cuidam em casa, não relataram o que faziam.

- 04 pessoas disseram que em qualquer lesão nas pernas ou pés, procuravam o médico. Elas já tinham sequelas das complicações do diabetes ("pé diabético").

- 03 pessoas afirmaram que nunca tiveram lesões ou calos nos pés e pernas e não disseram o que fariam caso os tivessem.

Em relação à ocorrência de lesão nas pernas ou pés, $68,8 \%$ dos entrevistados referiram que nunca tiveram qualquer tipo de lesão nas pernas ou pés,

$12,5 \%$ pessoas tiveram complicações nos pés e cuidaram em casa, sendo que destas, duas não haviam percebido a lesão, tendo notado-a quando já havia evoluído; $18,8 \%$ procuraram o médico logo ao sofrerem a lesão.

A maioria das pessoas $(68,8 \%)$ considerou-se segura quanto às informações que tem sobre sua doença para evitar futuras complicações.

Dos entrevistados, 59,4\% referiram necessidade de folhetos explicativos de fácil compreensão para adquirir mais conhecimentos sobre o diabetes e suas complicações, propiciando condições para melhorar sua qualidade de vida. Os temas referidos com maior frequência para a elaboração de material educativo foram: abordar o diabetes como um todo (10), alimentação (7), complicações (4) e aplicação de insulina (4), cuidados com os pés (3), controle do diabetes (2), exercícios (1) e uma não respondeu.

\section{DISCUSSÃO}

A maior parte da amostra tinha 50 anos de idade ou mais, o que era esperado tendo em vista que o Ambulatório atende basicamente clientes diabéticos do tipo 2.

A participação familiar é de fundamental importância no autocuidado do diabético, pois, muitas vezes, o cliente apresenta limitações para exercê-lo, tais como: retinopatia, ausência de membros, devendo um dos familiares ou mesmo a pessoa que o faz companhia aprender a executar os cuidados relativos à alimentação, medida da glicosúria e/ou aplicação de insulina, bem como manter a regularidade dos mesmo(13)

Foi grande o número de diabéticos entrevistados que referiram ser viúvos ou solteiros $(25 \%)$, mas só quatro $(12,5 \%)$ moram sozinhos, o que pode dificultar o tratamento.

Autores relatam que o fato de poder contar com o apoio da família (cônjuges ou filhos) é fundamental para o diabético, estimulando-o para a realização do autocuidado e auxiliando-o quando necessário (14).

É importante conhecer o grau de escolaridade do cliente diabético para planejar a atuação de forma correta, ou seja, facilitar a compreensão do mesmo em relação às informações sobre o diabetes. Tendo em vista a elevada percentagem de clientes sem escolaridade e com ensino fundamental incompleto na amostra estudada, será necessário utilizar métodos adequados, incentivando o autocuidado, visando a incorporação de novos conhecimentos, além de repensar o que já sabem, mudando sua forma de lidar com a doença e vivendo de uma maneira mais saudável. 
O que chama a atenção em relação à aposentadoria é que pouco mais da metade eram pessoas aposentadas, $53,1 \%$, embora $43,8 \%$ tivessem 60 anos ou mais de idade e a aposentadoria no Brasil venha ocorrendo em faixas etárias precoces ${ }^{(15)}$.

Embora a maioria da amostra tenha até 20 anos de doença, é importante ressaltar que os diabéticos com mais de vinte anos de duração da doença têm grandes chances de apresentar doença vascular periférica, sendo fundamental a identificação dos fatores de risco, além da orientação e discussão sobre como prevenir o aparecimento de complicações $(3,5,6)$

Os clientes que fazem uso de hipoglicemiante oral $-59,4 \%$, incluindo os que usam o hipoglicemiante e a insulina simultaneamente - devem estar cientes de que há vários tipos de medicamentos, com diferentes tempos de ação.

Dezesseis pessoas (50\%) faziam uso de insulina, o que implica em acesso à informação sobre os diferentes tipos do produto, tempo de ação e formas adequadas para a sua conservação; o modo correto de aplicação de insulina, os diferentes locais de aplicação, e aprender a fazer a mistura de diferentes tipos dessas substâncias. Devem ser informados, ainda, sobre a possibilidade de hipoglicemia, os sintomas e como preveni-los; o uso de fitas reagentes para sangue e urina para pesquisa de glicose, que auxiliam no controle do diabetes, o que é corroborado pelo Ministério da Saúde').

A atividade fisica foi referida somente por $40,6 \%$ dos entrevistados, sendo a mais freqüente a caminhada. O Ministério da Saúde preconiza a prática do exercício físico de forma regular - após avaliação das condições do cliente - de intensidade progressiva, duração adequada e modalidades variáveis, devendo fazer parte do esquema de tratamento dos diabéticos e ser praticado somente quando o estado metabólico estiver razoavelmente compensado. $\mathrm{O}$ tipo de exercício deve ser adequado às possibilidades e limitações do cliente, considerando-se as possiveis complicações crônicas do diabetes, como retinopatia, nefropatia, neuropatia, etc. Durante a prática da atividade física poderá ocorrer hipoglicemia e o cliente deve se alimentar antes do início da mesma, proporcionalmente a intensidade do exercício; o esquema terapêutico de insulina deve ser adaptado ao programa de atividade fisica ${ }^{(7)}$.

Embora a orientação para os diabéticos e seus familiares é que os pés precisam ser examinados diariamente, para detectar hiperemia, bolhas, calosidade ou ulceração, na amostra estudada $65,6 \%$ examinavam os pés, embora nem todos o fizessem diariamente. Para os clientes que possuem déficit visual, ou mobilidade diminuída das articulações (especialmente os mais idosos), o uso de um espelho para a inspeção das plantas dos pés ou para que outro membro da família os inspecione, pode ser necessário.
Estas orientações em relação aos cuidados com os pés são preconizadas por diferentes autores ${ }^{(6,7)}$.

É recomendado o uso de sapatos fechados confortáveis para maior proteção dos pés, pois muitas vezes, os diabéticos já têm a neuropatia periférica, que pode levar a diminuição da sensibilidade dolorosa e tátil dos pés, dificultando a percepção da ocorrência de uma lesão. E importante para evitar o aparecimento de lesões nos pés que o interior dos calçados seja inspecionado quanto a pontos em relevo ou presença de pequemos objetos, através de inspeção visual e manual, realizadas diariamente. $\mathrm{O}$ uso de sapatos inadequados pode propiciar a formação de bolhas e calosidades nos pés, o que poderá comprometer sua integridade, ocasionando lesões de continuidade que podem não ser percebidas, até que uma infecção grave tenha se desenvolvido.

A forma de cuidado das lesões nos pés referidas pelos entrevistados não é a recomendada no serviço estudado, pois na maioria dos casos, somente a limpeza do local com água e sabonete apresenta bons resultados.

O tipo de piso da residência do diabético é de extrema importância para a prevenção de acidentes em casa, relacionados à queda, que podem causar grandes traumas aos diabéticos que, muitas vezes, têm acentuada diminuição da acuidade visual. $\mathrm{Na}$ amostra estudada, a maioria das residências tinha piso de cerâmica $(46,9 \%)$ e o quintal era cimentado $(46,9 \%)$.

Mais da metade dos clientes $68,8 \%$, disseram sentir-se seguros quanto às informações que têm sobre sua doença para evitar futuras complicações, porém, levando-se em conta a análise dos dados, verificou-se que os mesmos não realizam os cuidados necessários para evitar as complicações. A resposta dada à questão poderia ser entendida como uma negação de sua condição, pois, ao mesmo tempo que relatam que estão informados sobre o diabetes, não se cuidam adequadamente.

A maioria da amostra $(59,4 \%)$ sente necessidade de folhetos explicativos, "aulas" de fácil compreensão para conhecer mais sobre o diabetes e suas complicações, visando uma melhor qualidade de vida.

\section{CONCLUSÃO}

Este estudo permitiu avaliar o conhecimento do cliente diabético em relação aos cuidados com os pés, considerando-se tanto o aprendizado que o mesmo adquiriu em consultas junto à equipe multidisciplinar como experiências anteriores.

A maioria das pessoas entrevistadas $(59,4 \%)$ era do sexo feminino, sendo as faixas etárias predominantes de 50 a 59 anos $(43,8 \%)$ e acima de 60 $(43,8 \%)$. 
Em relação ao estado civil, $75 \%$ eram casadas. Quanto à escolaridade, $65,6 \%$ possuíam o ensino fundamental incompleto; $53,1 \%$ dos entrevistados eram aposentados; $71,9 \%$ eram diabéticos há menos de 10 anos, e a maioria fazia uso de hipoglicemiante oral para o tratamento do diabetes.

Dos entrevistados, $87,5 \%$ moravam em casa própria e $71,9 \%$ moravam com o cônjuge / cônjuge e filhos.

A alteração na acuidade visual foi referida por $40,6 \%$. Somente $40,6 \%$ dos entrevistados realizavam algum tipo de atividade fisica (sendo predominante a caminhada) .

O uso de sapato fechado foi referido por $62,5 \%$, o que é recomendado no serviço estudado.

A maioria dos entrevistados $(81,3 \%)$ sabia que os cuidados adequados com os pés eram importantes para prevenir as complicações pertinentes ao diabetes.

Os temas referidos com maior freqüência pelos entrevistados para a elaboração de material educativo foram, por ordem de freqüência: abordar o diabetes como um todo, alimentação, complicações e aplicação de insulina, cuidados com os pés, controle do diabetes e exercícios.

As ações educativas deveriam estar disponibilizadas para todos os clientes do Ambulatório de Diabetes e seus familiares, tendo como referência a criação de vínculo com os profissionais / Serviço, o que poderá auxiliar na aderência ao tratamento, além de informá-los sobre os cuidados com o diabetes em geral e com os pés, em particular.

Concluiu-se que os clientes sabiam que os cuidados adequados com o pé eram necessários para evitar as complicações, porém o autocuidado não era realizado corretamente. Para que isso seja incorporado em seu cotidiano, é fundamental que tenham acesso às informações sobre diabetes, juntamente com seus familiares, além de habilidade e motivação.

Consideramos que é fundamental que nas práticas educativas exista uma troca entre o cliente, os profissionais de saúde e a família, buscando a construção da cidadania e a qualidade de vida.

\section{REFERÊNCIAS BIBLIOGRÁFICAS}

(1) Brasil. Ministério da Saúde. Sociedade Brasileira de diabetes. Programa Harvad/Joslin/SBD. Diabetes Mellitus. Guia básico para diagnóstico e tratamento. Brasília: Ministério da Saúde; 1996.

(2) Zanetti ML, Mendes IAC. Tendência do locus de controle de pessoas diabéticas. Rev Esc Enferm USP 1993 agosto; 27(2): 246-62.

(3) Franco LJ. Bases epidemiológicas para o controle do Diabetes mellitus. In: Costa MFFL, Sousa RP, organizadores. Qualidade de vida: compromisso histórico da epidemiologia. Belo Horizonte: COOPMED / ABRASCO; 1994. p.79-86-

(4) Boulton AJ. Why bother educating the multidisciplinary team and the patient - the example of prevention of lower extremity amputation in diabetes. Patient Educ Couns 1995; 26(1/3): 183-8.

(5) American Diabetes Association. Preventive foot care in people with diabetes. Diabetes Care 1999; 22(sup.1): S54-5.

(6)Gamba MA. A importância da assistência de enfermagem na prevenção, controle e avaliação a pacientes portadores de diabetes com neuropatia e vasculopatia. Acta Paul Enferm $1991 ; 4(2 / 4): 7-19$

(7) Brasil. Ministério da Saúde. Secretaria de Assistência à Saúde. Departamento de Assistência e Promoção à Saúde. Coordenação de Doenças Crônico-Degenerativas. Manual de diabetes. 2" ed. Brasília: Ministério da Saúde; 1993.

(8) Smeltzer SC, Bare BG. Brunner \& Suddarth: Tratado de enfermagem médico-cirúrgica. $7^{\prime \prime}$ ed. Rio de Janeiro: Guanabara-Koogan; 1994. v.2.

(9) Cocco MIM. A ideologia do enfermeiro: prática educativa em saúde coletiva. [dissertação] Campinas (SP): Faculdade de Educação da Universidade Estadual de Campinas; 1991-

(10) Basílio M, Vilela MD, Roque S. O pé diabético nos caminhos da cirurgia. Nursing, Lisboa 1997; 9(108):34-8.

(11) Cocco MIM. Práticas educativas em saúde e a construção do conhecimento emancipatório. In: Bagnato MHS, Cocco MIM, Sordi, MRL, organizadoras. Educação, saúde e trabalho: antigos problemas, novos contextos, outros olhares. Campinas: Alínea; 1999. p. 63-70.

(12) Minayo MCS. O desafio do conhecimento: pesquisa qualitativa em saúde. $3^{\circ}$ ed. São Paulo/ Rio de Janeiro: Hucitec/Abrasco; 1994.

(13) Luce M., Padilha MICS, Almeida RLV, Silva MO. O preparo para o autocuidado do cliente diabético e família. Rev Esc Enferm USP 1991; 25(2):137-52.

(14) Marocco EC, Cocco MIM. Práticas educativas em diabetes mellitus: proposta para elaboração de material pedagógico. Relatório Final de Pesquisa PIBIC-CNPq. Campinas: Universidade Estadual de Campinas; 1998.

(15) Cocco MIM, Monteiro MS. Aging and work in development countries -- the case of Brazil. In: Program \& Abstracts do $2 \mathrm{n}^{\mathrm{d}}$ International ICOH Conference on Aging and Work; 1998 sept. 14-16; Elsinore / Copenhague. Copenhague: ICOH, 1998. p. 66

\section{Artigo recebido em 05/01/00}

\section{Artigo aprovado em 14/08/02}

